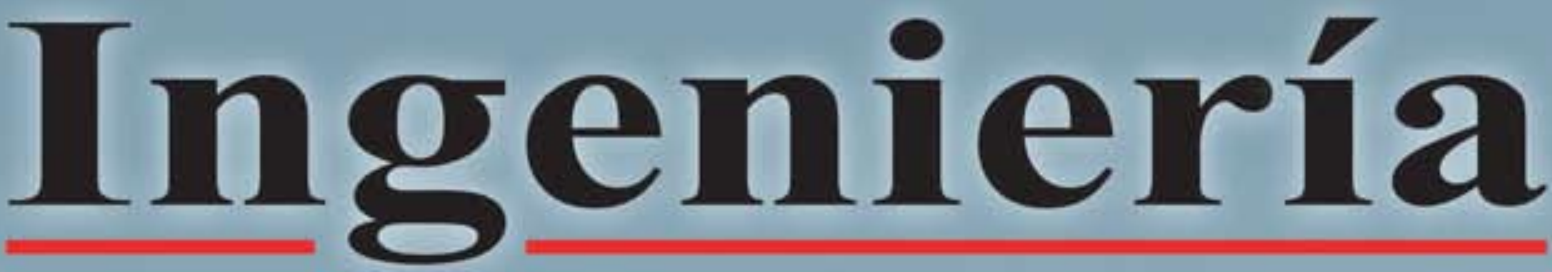

ENERO/DICIEMBRE 2004 - VOLUMEN 14 - N¹ y 2

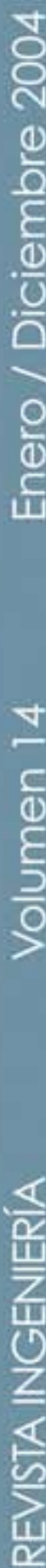
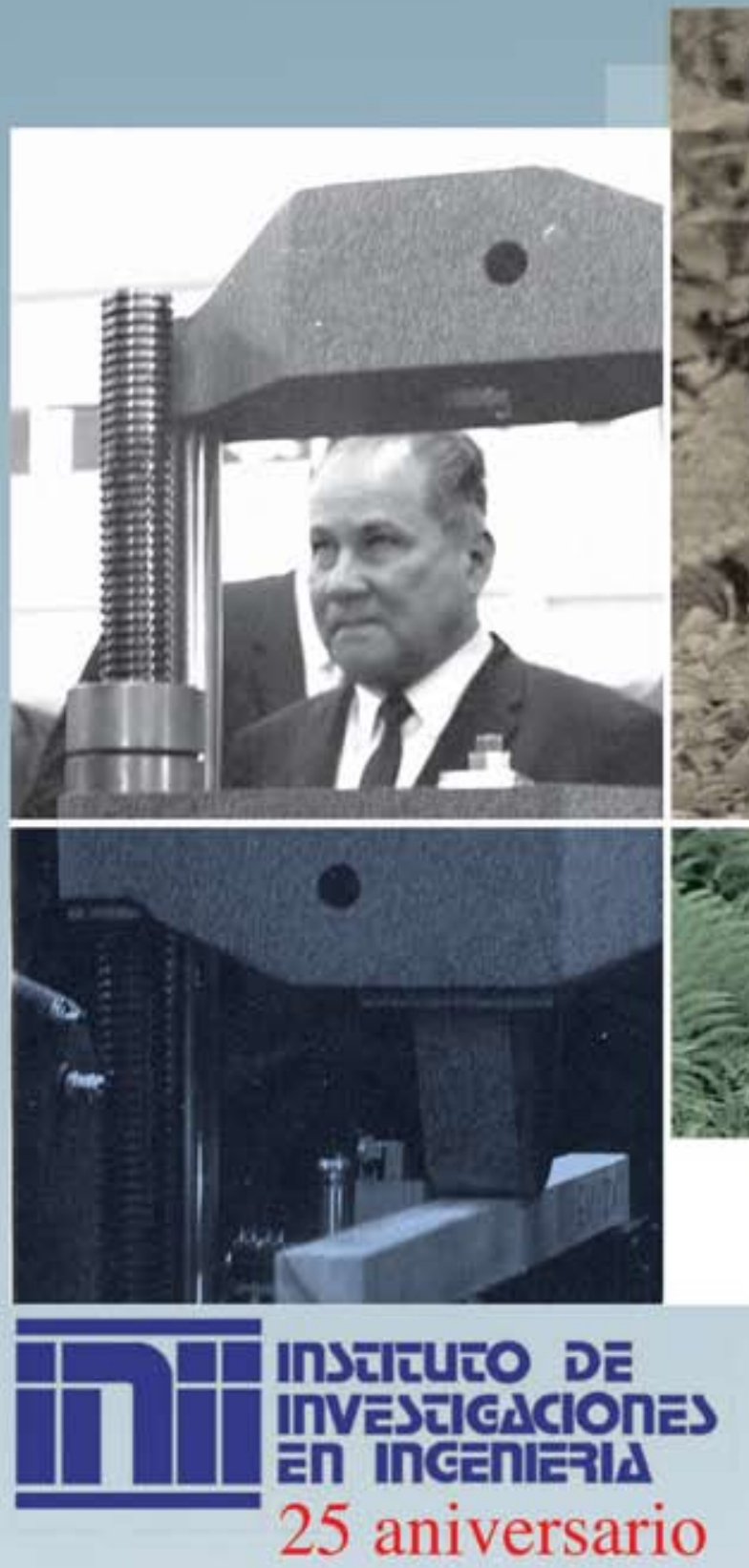

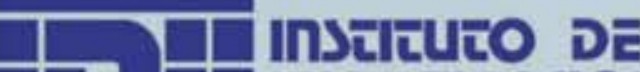
T W Investcscines हn Incenlei|d 25 aniversario

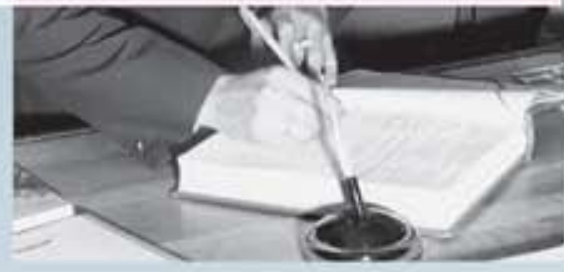




\title{
CÓDIGO SÍSMICO DE COSTA RICA VERSIÓN 2002. CONSIDERACIONES ENERGÉTICAS PARA LOS ESPECTROS DE DISEÑO DEL ÁREA METROPOLITANA
}

\author{
Rubén Leandro Ulloa \\ Guillermo Santana Barboza
}

\begin{abstract}
Resumen
Se presenta el tercero y último informe de investigación para validar las formas espectrales que se utilizan para el diseño sismorresistente de las estructuras civiles definidas en la nueva versión del Código Sísmico de Costa Rica (CSCR-2002). Con el uso de una base de datos de registros sísmicos, nacionales y extranjeros, se realizan 62900 corridas de sistemas de un grado de libertad para determinar los espectros de energía, en cada uno de los escenarios sísmicos obtenidos en el Área Metropolitana, para todos los valores de ductilidad. Las formas espectrales del CSCR2002 inducen bajas velocidades energéticas a las estructuras, lo que corresponde a posibles desaciertos a la hora de aproximar los niveles de daño esperado en las estructuras.
\end{abstract}

Palabras clave: espectros de energía, ductilidad constante, espectros de diseño elástico, espectros de diseño inelástico, diseño sísmico, sistemas de un grado de libertad, energía absoluta, energía relativa, velocidad energética equivalente, índice energético.

\begin{abstract}
This is the third and last report of a series of three investigations whose objective is to validate the spectral shapes that are using for the seismic-resistant design of the buildings defined into the new version of the Seismic Code of Costa Rica (CSCR-2002). With the use of a database of seismics records including national ones and foreign ones, have been made 62900 analysis of single degree of freedom to determinate the energy spectra in according with each seismic scenary obtained in the Metropolitan Area of Costa Rica through the ductilities values. The spectral shapes of the CSCR-2002 are inducing low energetical velocities to the structures, corresponding a potential mis takes in order to approach the damage level expected in the structures.
\end{abstract}

Keywords: energy spectra, constant ductility, elastic design spectra, inelastic design spectra, seismic design, seismic design code, single degree of freedom, absolutely energy, relative energy, equivalent energetic velocity, energetic index.

Recibido 08-VII-05 • Aceptado 14-X-05

\section{INTRODUCCIÓN}

El Área Metropolitana presenta la mayor densidad de población en Costa Rica. Cualquier análisis tendiente a definir espectros de diseño para esta región, conlleva un riesgo de incertidumbre asociado, debido a la magnitud de los posibles daños que pueden ocurrir.

Además del aspecto tradicional de definir los espectros de resistencia aplicables para el diseño de estructuras, es adecuado conocer la forma como la energía del sismo es absorbida y asimilada por las edificaciones. El dimensionar la estructura de acuerdo con este patrón energético de energía absorbida, liberada y disipada, permite ampliar el panorama de conocimiento del diseño sismorresistente.

\section{MODELO ESTRUCTURAL}

Consiste en un sistema de un grado de libertad, representado por un marco estructural formado por dos columnas con masa despreciable de idéntica sección y rigidez (con rigidez infinita 
en el sentido vertical y flexibles en el sentido horizontal, susceptibles a deformaciones laterales) las cuales se encuentran unidas en su parte superior por medio de un piso infinitamente rígido, el cual tiene toda la masa distribuida de manera uniforme en su longitud. Adicionalmente, para tomar en cuenta la disipación de energía se cuenta con un amortiguador viscoso.

El modelo de comportamiento de material que se va a adoptar en el análisis es el bilineal, el valor de la rigidez secundaria o rigidez postfluencia, para efectos de este trabajo, será de $10 \%$ del valor de la rigidez inicial o rigidez elástica.

\section{3. ÁREA METROPOLITANA DE COSTA RICA}

El análisis se especifica a la zona de mayor desarrollo urbano, correspondiente a la denominada Gran Área Metropolitana. Esta zona comprende la depresión central localizada en la parte sur de la Cordillera Volcánica Central y al este de la Cordillera de Talamanca. Dentro de esta área se ubica la mayor parte de la población costarricense, asentada sobre las ciudades de San José, Cartago, Heredia, Alajuela, Turrialba, San Ramón entre las más importantes.

Los cuatro tipos de suelos definidos por el CSCR$2002\left(S_{1}, S_{2}, S_{3}\right.$ y $\left.S_{4}\right)$ se localizan dentro del Área Metropolitana, sin embargo para efectos del presente trabajo no va a considerarse el perfil de suelo muy blando. Esto por razones de ser el perfil de suelo con la menor presencia porcentual dentro de la región y por sus características tan diferentes con respecto al suelo blando.

Como forma de comprobación y para asegurar un mejor detalle de profundidad del análisis, se especifica para el Área Metropolitana una base de registros sísmicos, referida exclusivamente a sismos de mecanismo de falla lateral (coincidente con las fallas transcurrentes que caracterizan la mayor influencia sísmica de esta zona) en zona sísmica $\mathrm{Z3}$ para tres tipos de suelo de cimentación $\left(\mathrm{S}_{1}, \mathrm{~S}_{2}\right.$ y $\left.\mathrm{S}_{3}\right)$. El rango de períodos comprende desde $0,1 \mathrm{~s}$ hasta $1,4 \mathrm{~s}$, el cual corresponde al rango esperado para la mayoría de las edificaciones del Área Metropolitana, ya que con pocas excepciones no existen estructuras muy flexibles.

La base registros sísmicos está constituida por un total de 16 registros (la combinación de zona sísmica Z3 y tipo de suelo $\mathrm{S}_{1} \mathrm{Z} 3 \mathrm{~S} 1$ tiene 4 registros y las zonas $\mathrm{Z3}_{3} \mathrm{~S}_{2}$ y $\mathrm{Z3}_{3} \mathrm{~S}_{3}$ tienen 6 registros cada una de ellas). Todos son sismos con mecanismo de falla lateral con valores de aceleraciones pico efectivas dentro del rango de $0,2 \mathrm{~g}$ hasta $0,3 \mathrm{~g}$. Todos los registros sísmicos corresponden a eventos extranjeros, ya que no fue posible localizar eventos nacionales con valores de EPA correspondientes a la zona sísmica Z3.

El Cuadro 1 presenta las características de los sismos de la base de datos.

Cuadro 1. Características de los eventos sísmicos de la base de registros

\author{
Evento sísmico, país \\ Morgan Hill, Estados Unidos \\ Coyote Lake, Estados Unidos \\ Patra, Grecia \\ Imperial Valley, Estados Unidos \\ South Iceland, Islandia \\ Adana, Turquía \\ Catania, Italia \\ Parkfield, Estados Unidos \\ Izmit, Turquía \\ Erzincan, Turquía
}

Fecha
Magnitud Profundidad (km)

$\begin{array}{ll}\mathrm{MS}=6,1 & -- \\ \mathrm{MS}=5,7 & -- \\ \mathrm{MS}=6,4 & 13 \\ \mathrm{MS}=6,3 & 16 \\ \mathrm{MS}=7,1 & 6,3 \\ \mathrm{MS}=6,2 & 32 \\ \mathrm{MS}=5,8 & 15 \\ \mathrm{MS}=6,4 & 10 \\ \mathrm{Mw}=7,4 & 17 \\ \mathrm{MS}=6,8 & 16\end{array}$

--
--
13
16
6,3
32
15
10
17
16

Mecanismo focal

$$
\begin{gathered}
24 \text { de abril de } 1984 \\
08 \text { de junio de } 1979 \\
14 \text { de julio de } 1993 \\
19 \text { de mayo de } 1940 \\
17 \text { de junio del } 2000 \\
27 \text { de junio de } 1998 \\
13 \text { de diciembre de } 1990 \\
28 \text { de junio de } 1966 \\
19 \text { de agosto de } 1999 \\
19 \text { de marzo de } 1992
\end{gathered}
$$

$$
\begin{gathered}
\text { Lateral } \\
\text { Lateral } \\
\text { Lateral } \\
\text { Lateral derecho } \\
\text { Lateral } \\
\text { Lateral } \\
\text { Lateral } \\
\text { Lateral derecho } \\
\text { Lateral }
\end{gathered}
$$

Lateral derecho 


\section{USO DE LA ENERGÍA COMO CRITERIO DE DISEÑO SISMORRESISTENTE}

La ecuación básica de diseño sismorresistente establece que el sistema estructural debe poseer una capacidad de respuesta por lo menos igual a la cantidad demandada. Esto aplica para resistencia, desplazamiento y energía. Tradicionalmente, la ductilidad de desplazamiento ha sido usada como el criterio que permite establecer el espectro de diseño inelástico, a partir del cual se obtiene la resistencia máxima requerida o capacidad de resistencia, la cual debe ser provista por la estructura para permitir el cumplimiento de la ecuación básica de diseño. Pero, el criterio de diseño basado en fuerzas o desplazamientos, no es suficiente para expresar el potencial de daño de los movimientos del terreno, ya que el potencial de daño no depende exclusivamente del desplazamiento máximo y el daño acumulativo, depende directamente del efecto de la duración y los ciclos de carga de la estructura, y estos factores no son obtenidos ni deducidos a partir de la ductilidad de desplazamiento.

Como alternativa de diseño se establece la definición de las ecuaciones de energía, bajo la premisa de incluir, dentro de ellas, a los factores que tienen relevancia sobre el potencial de daño de las estructuras, ya que en principio todo sistema estructural, antes de regirse por fuerzas o desplazamientos, debe ser comprendido como un sistema que tiene la capacidad de absorber y disipar energía, y precisamente de la velocidad con la cual llegue a cumplir esta absorción o disipación, depende su integridad estructural. El evento sísmico es un fenómeno que proporciona energía a la estructura y ésta, en conjunto con su interacción con el sitio de cimentación, debe asimilar dicho patrón energético y por medio de deformaciones internas y desplazamientos externos, proceder a evitar el colapso, siempre y cuando el detallado y dimensionado estructural sea el adecuado.

Partiendo del modelo idealizado del sistema de un grado de libertad, el problema de la acción de un movimiento sísmico sobre una estructura puede ser analizado desde dos puntos de vista. El primero corresponde a suponer una acción o efecto directo sobre la base de la estructura, lo que implica que el modelo estructural debe considerarse con capacidad de movilizarse como cuerpo rígido y luego presentar deformaciones relativas internas, dicho enfoque se denomina sistema de base móvil. El otro punto de vista consiste en suponer que la acción del movimiento sísmico debe traducirse en una fuerza equivalente lateral aplicada en la parte superior del modelo, lo que significa la ausencia total de desplazamiento de cuerpo rígido y todo el movimiento se considera relativo dentro del sistema.

Los dos puntos de vista son válidos y conducen exactamente al mismo concepto matemático de la siguiente ecuación

$E_{\text {entrada }}=E_{\text {cinética }}+E_{\text {restauración }}+E_{\text {histeréstica }}+E_{\text {amortiguación }}$

Además de conducir al mismo lenguaje de cálculo, ambos enfoques conducen a la misma conclusión: la energía de entrada debe ser numéricamente igual a la suma de la energía cinética, la energía elástica de deformación recuperable, la energía plástica histerética irrecuperable y la energía de amortiguamiento viscoso. Entre los componentes de la energía de entrada hay factores que suman para la absorción de la energía y otros que suman para la disipación de la energía.

Se considera Energía absorbida a la suma de la energía de restauración y la energía histerética. Se considera Energía disipada a la suma de la energía cinética y la energía de amortiguamiento. Así entonces, en cualquier instante de tiempo, la Energía de entrada es igual a la acción simultánea de una Energía absorbida y una Energía disipada, y ambas dependen de las características estructurales del sistema.

Sin embargo, sus resultados son diferentes, ya que se basan en una característica muy importante: la elección del desplazamiento total 
del sistema dependiente del tiempo, en función de la representación del efecto del movimiento del terreno.

El método utilizado en este análisis es la energía absoluta (sismo actuando sobre la base móvil del modelo) donde define el desplazamiento total del sistema como la suma de dos componentes: el desplazamiento del terreno debido al movimiento sísmico y el desplazamiento relativo de la masa del modelo con respecto al terreno. Esto fundamentado en dos razones: el desplazamiento relativo se relaciona con el parámetro de daño estructural, por lo que es recomendable utilizar la energía absoluta para períodos muy cortos y la energía relativa para períodos muy largos (en este caso, el rango de períodos no llegará a la zona de estructuras muy flexibles) y además la energía absoluta representa la energía física real de entrada al sistema.

Adicionalmente, se aprovecha la similitud numérica existente entre ambos métodos para el rango de períodos de $0,5 \mathrm{~s}$ hasta $4,0 \mathrm{~s}$, y la similitud también existente para regiones cercanas a los períodos predominantes de la excitación sísmica.

La siguiente expresión matemática corresponde a la derivación de la energía de entrada absoluta, a partir de la ecuación del movimiento.

$$
\begin{aligned}
& \frac{m \dot{u}_{1}^{2}}{2}+\int c \dot{u} d u+\int f_{x} d u=\int m \ddot{u}_{1} d u \\
& u_{1}=u_{g}+u \\
& E_{i}=\int m \ddot{u}_{t} d u_{g} \text { Energía de entrada absoluta }
\end{aligned}
$$

Suponiendo estructuras muy rígidas (períodos que tienden a cero) y estructuras muy flexibles (períodos que tienden a infinito) se obtienen las siguientes relaciones.

$$
\begin{array}{ll}
T \rightarrow \infty & T \rightarrow 0 \\
\frac{E_{\text {entradaabsoluta }}}{m}=0 & \frac{E_{\text {entradaabsoluta }}}{m}=\frac{\dot{u}_{g}^{2}}{2} \\
\frac{E_{\text {entradarelativa }}^{\prime}}{m}=\frac{\dot{u}_{g}^{2}}{2} & \frac{E_{\text {entradarelativa }}}{m}=0
\end{array}
$$

\section{VELOCIDADES ENERGÉTICAS EQUIVALENTES E ÍNDICES ENERGÉTICOS}

Es interesante conocer si las formas de los espectros de diseño del Código conducen a comportamientos de las estructuras tendientes a presentar tasas de absorción y disipación de energía, que no correspondan a lo esperado, de acuerdo con los propios registros.

Al ser la energía de entrada absoluta una representación del trabajo hecho por el cortante total de la base sobre el desplazamiento de la fundación, existe una relación de las tasas o velocidades de entrada de energía a la estructura con el daño potencial que pueda sufrir la estructura. (Fajfar, 1995), (Uang \& Bertero, 1990) y (Uang \& Bertero, 1988). El desconocimiento de la velocidad de arribo de la energía (sea energía de entrada, energía de absorción o energía de disipación) puede conducir a interpretaciones erróneas en cuanto a la formación de mecanismos inelásticos que no corresponden a la realidad.

Dicha idea se refleja en las velocidades espectrales equivalentes (columna izquierda de la ecuación 4) como índices cualitativos de la demanda energética en un sistema estructural. Estas velocidades son artificios que relacionan las energías (de entrada, absorbida y disipada) en términos de velocidades equivalentes, tal y como se muestra en la siguiente ecuación:

$$
\begin{aligned}
& V_{\text {disipada }}=\sqrt{2 \frac{E_{\text {disipada }}}{m}} \quad I E_{\text {disipada }}=\frac{1}{T_{\max }} \int_{0}^{T_{\max }} V_{\text {disipada }} d T \\
& V_{\text {absorbida }}=\sqrt{2 \frac{E_{\text {absorbidada }}}{m}} \quad I E_{\text {absorbida }}=\frac{1}{T_{\max }} \int_{0}^{T_{\max }} V_{\text {absorbida }} d T \\
& V_{\text {entrada }}=\sqrt{2 \frac{E_{\text {entrada }}}{m}} \\
& I E_{\text {entrada }}=\frac{1}{T_{\max }} \int_{0}^{T_{\max }} V_{\text {entrada }} d T
\end{aligned}
$$

La velocidad espectral equivalente de energía absorbida se ve afectada por el efecto de sitio. Además es considerado un índice de demanda de energía, ya que converge a la pseudovelocidad para el caso elástico; y es una cantidad estable para estimar la demanda de energía en los sistemas dúctiles. 
El índice energético (IE en la ecuación 4) representa la velocidad equivalente de energía demandada en promedio por sistemas de un grado de libertad sometidos a movimiento del terreno. Se encuentra correlacionado con el valor de la aceleración máxima del terreno, la razón espectral V/A, la duración efectiva y el contenido de frecuencias, lo cual lo convierte en una medida representativa del potencial de daño

La energía absorbida es un índice estable para representar la demanda de energía en sistemas de un grado de libertad, ya que converge al valor de la pseudovelocidad en el caso elástico el cual, en la forma de pseuodoaceleración, es un parámetro de diseño.

\section{ESPECTROS DE ENERGÍA PARA DUCTILIDAD CONSTANTE}

Se realiza la comparación de los resultados obtenidos con la base de registros con respecto a los valores del CSCR-2002. Desde la Figura 1 hasta la Figura 12 se muestran, de forma gráfica, estas relaciones.

Para el caso de registros en el tipo de suelo $\mathrm{S}_{1}$, la ubicación de la zona de discrepancia con el CSCR-2002 se traslada hacia la zona de períodos largos en todas las ductilidades. $\mathrm{La}$ zona de períodos intermedios ahora pasa a ser la región en la cual existe poca discrepancia, mientras la zona de períodos cortos, mantiene su comportamiento.

Para el caso de registros en el tipo de suelo $\mathrm{S}_{2}$ se nota un comportamiento similar, con un desplazamiento de la principal zona de discrepancia de la región de períodos intermedios a la región de períodos largos. La zona de períodos cortos permanece prácticamente invariable, pero existe, además del traslado a la región de períodos largos de la zona de discrepancia, un incremento bastante notorio en cuanto a la magnitud de las diferencias. Esta situación ocurre en todas las ductilidades.
Para el caso de registros en suelo $\mathrm{S}_{3}$, ocurre un incremento importante de los valores de discrepancias con el CSCR-2002, a tal punto que prácticamente en todas las zonas en las cuales el espectro de este código presentaba los valores mayores, ahora se convierten en zonas en donde la base de datos presenta valores mayores, implicando así que la forma espectral del código CSCR-2002 subvalora la solicitación real.

\section{COMPARACIÓN CON EL CÓDIGO SÍSMICO DE COSTA RICA VERSIÓN 2002 EN EL ÁREA METROPOLITANA}

Con un valor de amortiguamiento del $5 \%$ se obtienen los espectros de resistencia. Para el caso de la base de datos son utilizados los valores del promedio más la desviación estándar $(84,1$ percentil) los cuales se comparan con los valores medios obtenidos del CSCR-2002, los que tienen ya la consideración de la desviación estándar, debido al uso del criterio de Riddell y Newmark. Esto permite encontrar zonas en donde existan discrepancias numéricas entre estos valores.

Se presenta la comparación de las velocidades espectrales equivalentes y los índices energéticos para las demandas de energía de entrada, absorbida y disipada, para el caso de las combinaciones en el Área Metropolitana (Zona Sísmica Z3). Valores mayores de la base de registros sísmicos con respecto a los valores obtenidos con las formas espectrales del CSCR-2002 tienden a interpretarse como una deficiencia de parte de esta normativa para expresar la correcta velocidad de la demanda de energía. Si esto se encuentra relacionado con los índices de daño, existe la posibilidad de conducir a diseños erróneos a la hora de tomar en cuenta las incursiones inelásticas de una estructura.

La forma de interpretar las velocidades equivalentes es relevante, ya que al presentarse las tres velocidades equivalentes en un mismo gráfico, no implica que se estén representando, de forma simultánea y en términos absolutos, las energías sino solamente las velocidades, esto 
significa que aunque una velocidad prevalezca sobre las otras dos, no implica que dicha energía sea mayor que las otras dos, únicamente es mayor su velocidad de incorporación al sistema.

Para el perfil de suelo rocoso $\left(S_{1}\right)$ : en todos los valores de ductilidad siempre el valor de las velocidades equivalentes energéticas de la base de registros sísmicos es superior al valor obtenido de las formas espectrales del CSCR2002. La tendencia de comportamiento para períodos cortos, en el caso de la base de registros, es presentar el mayor valor de la velocidad equivalente de energía disipada, seguido por la velocidad equivalente de energía de entrada y la velocidad equivalente de energía absorbida, presentando el menor valor absoluto.

En el caso del CSCR-2002 para períodos cortos se mantiene esta tendencia para valores de ductilidad menores a 3. A partir de esta ductilidad, la velocidad equivalente de la energía de entrada sobrepasa a la velocidad equivalente de energía absorbida $\mathrm{y}$, conforme aumenta el valor de la ductilidad, aumenta también el rango de períodos en los cuales ocurre esta situación, para finalmente en el valor de ductilidad 6, presentar la velocidad equivalente de energía de entrada valores mayores en todo el rango de períodos. Para todos los valores de ductilidad y todos los períodos, siempre la velocidad equivalente de la energía absorbida presenta el menor valor absoluto. A partir del valor de ductilidad 1,5 las curvas se cruzan en cierto punto, que coincide con los $1,1 \mathrm{~s}$ para el valor de ductilidad $1,5 \mathrm{y}$ se va reduciendo hasta $0,3 \mathrm{~s}$ para ductilidad 6 .

El comportamiento de la base de registros es diferente, ya que la intersección de las velocidades equivalentes ocurre a partir del valor de ductilidad 3 , en valores de períodos cercanos a 1,15 s hasta llegar a 0,7 s para la ductilidad 6. Así, entonces el CSCR-2002 induce en las estructuras, para todos los valores de ductilidad, bajas velocidades equivalentes de energía disipada para las regiones de períodos intermedios, mientras que produce un incremento rápido de la velocidad equivalente de la energía de entrada, al ir aumentando el valor de la ductilidad. El comportamiento de la base de registros es similar, solo que presenta un desfase en el rango de períodos y con magnitudes de velocidades mayores.

Los índices energéticos en el caso del CSCR2002 para todos los valores de ductilidad, con excepción del caso elástico, presenta curvas casi paralelas entre ellos y tendencia asintótica individualmente a un valor constante a partir del valor de ductilidad 4. Para la base de registros existe el mismo incremento con respecto al caso elástico en el valor de ductilidad 1,5, continuando con una pendiente negativa y convirtiéndose luego en líneas paralelas y asintóticas a un valor constante a partir del valor de ductilidad 5. En ambos casos, dicho comportamiento se puede referir a niveles constantes de potencial de daño para valores grandes de ductilidad.

Para el perfil de suelo firme $\left(S_{2}\right)$, la presencia del suelo firme acelera la intersección de las velocidades equivalentes, tanto para la base de registros como para las formas espectrales del CSCR-2002. Inclusive, desde valores de ductilidades tan bajos como 1,5, ya existe una marcada superioridad numérica de la velocidad equivalente de energía de entrada sobre la velocidad equivalente de energía disipada. En todos los valores de ductilidad, las estructuras están recibiendo de forma más rápida la energía que su capacidad de disiparla. Por esto, existe un valor importante de la velocidad equivalente de energía absorbida, que sirve para tomar la diferencia de energía que no es disipada, lo que se encuentra en relación directa con los índices de daño.

Comparando el índice energético de suelo firme con el correspondiente a suelo rocoso, existe la misma tendencia con la característica de no ser comportamiento asintótico, sólo se pierde el carácter asintótico ya que, a partir del valor de ductilidad 4 para el CSCR-2002 y a partir del valor de ductilidad 3 en la base de registros, las líneas no son horizontales y tienen pendiente positiva para el CSCR-2002 y pendiente negativa para la base de registros. 
Los valores de las velocidades equivalentes de todas las energías del CSCR-2002, en todo el conjunto de valores de ductilidades, son menores a los correspondientes de la base de registros sísmicos.

Para el perfil de suelo blando $\left(S_{3}\right)$, se acentúa el comportamiento observado en el caso del suelo firme. La intersección de las velocidades equivalentes sigue la misma tendencia, pero se aumentan las brechas entre las velocidades de forma más rápida a partir del valor de ductilidad de 3. Es importante el papel que juega ahora la velocidad equivalente de energía absorbida, superando a la velocidad equivalente de la energía disipada.

A partir del valor de ductilidad 4, no existe intersección de las líneas de velocidades equivalentes, únicamente ocurre en la región de los períodos cortos.

Como tendencia generalizada en todos los valores de ductilidad, el CSCR-2002 presenta magnitudes menores de las velocidades equivalentes energéticas con respecto a los valores de la base de registros sísmicos. Un comportamiento particular se nota con los índices energéticos, al ocurrir intersecciones entre las curvas de las velocidades equivalentes de energía disipada y de la energía absorbida, para valores de ductilidad mayores a 4. Esto aplica para el CSCR-2002 y para la base de registros. Esto puede ser debido a un potencial mayor de daño para estructuras de ductilidades altas en perfiles de suelo blando, lo que implica un necesario y especial cuidado en el detallado de las uniones estructurales.

\section{CONCLUSIONES}

Es necesario realizar estudios más detallados, con bases de datos más completas, para lograr un grado razonable de validación de los espectros de diseño y poder cumplir con el balance requerido entre seguridad estructural y costo económico de la edificación.
1. El diseño sismorresistente se ha efectuado tradicionalmente tomando como fundamento los espectros de respuesta de aceleración, velocidad y desplazamiento, como funciones directas de los valores máximos de dichos parámetros para cada período estructural. Esto limita el conocimiento de la información a únicamente los valores picos. El espectro de energía se convierte en una metodología de diseño de reciente desarrollo, que contempla toda la información de la historia de respuesta de un sistema de un grado de libertad. Permite la consideración de manera sencilla de los efectos de la duración del sismo en el diseño, representado en aspectos de la degradación de la rigidez, deterioro de la resistencia y aumento progresivo de la deformación.

2. Un método no tradicional de análisis de espectros de energía es concebido a partir de la fijación de las formas de los espectros de diseño del CSCR-2002, a diversas historias de aceleraciones en el tiempo (registros sísmicos) que determinan los valores máximos de absorción y disipación de energía por medio de dos parámetros: velocidades equivalentes energéticas e índices equivalentes energéticos, los cuales son obtenidos de los tradicionales sumandos de la ecuación de equilibrio energético, y permiten obtener una visión más específica en cuanto al comportamiento de la estructura ante la llegada de la energía sísmica.

3. La energía absorbida por un sistema estructural contempla la energía histerética. Dicha energía es considerada un parámetro relacionado con el potencial de daño estructural. De acuerdo con los registros sísmicos de la base de datos, existe una tendencia de valores fuertes de velocidad equivalente de energía absorbida para prácticamente todo el rango de períodos de estudio $(0,4 \mathrm{~s}$ hasta $1,4 \mathrm{~s})$ a partir del valor de ductilidad 4 en perfiles de suelo blando. Dicha tendencia no es evidenciada por el CSCR-2002, lo que puede provocar altos niveles de daño que no son contemplados a la hora de utilizar las formas espectrales del CSCR-2002. Es necesario estudiar dicha situación. 
4. En el Área Metropolitana, el CSCR-2002 obliga a que las estructuras civiles tengan velocidades bajas de demanda energética (para cualquiera de las energías de entrada, absorción o disipación), en todos los perfiles de suelo y en todos los períodos y ductilidades. Así, cualquier diseño estructural que utilice los espectros del CSCR-2002 e intente contemplar el efecto de la carga cíclica sobre la edificación, va a subestimar el verdadero comportamiento. Además, existe una fuerte evidencia de potenciales importantes de daño para estructuras muy dúctiles en perfiles de suelo blando, lo que amerita especial cuidado en su diseño estructural.

\section{REFERENCIAS BIBLIOGRÁFICAS}

Chou, Ch. \& Uang, Ch. (2000). Establishing absorbed energy spectra -an attenuation approach. Earthquake Engineering and Structural Dynamics, 29, 1441-1455.

Colegio Federado de Ingenieros y Arquitectos, (2002). Gutiérrez, J. (editor). Código Sísmico de Costa Rica. Versión 2002. Cartago: Editorial Tecnológica de Costa Rica.

Decanini, L. \& Mollaioli, F. (1998). Formulation of elastic earthquake input energy spectra. Earthquake Engineering and Structural Dynamics, 27, 1503-1522.

Malhotra, P. (2002). Cyclic-demand spectrum. Earthquake Engineering and Structural Dynamics, 31, 1441-1457.

Manfredi, G. (2001). Evaluation of seismic energy demand. Earthquake Engineering and Structural Dynamics, 30, 485-499.

Fajfar, P. (1995). Elastic and inelastic design spectra. En: European Conference on Earthquake Engineering (pp.1169-1178). Balkema, Rotterdam.
Ochi, K. \& Kondo, H. Energy input rate spectra of earthquake ground motions. En: 10th European Conference on Earthquake Engineering (pp. 641-644). Balkema, Rotterdam.

Santana, P. \& Terán-Gilmore, A. (1994). Damage potential of earthquake ground motions recorded in southern Central America. Revista Ingeniería de la Universidad de Costa Rica, 4(2), 75-88.

Sucuoglu, H. \& Nurtug, A. (1995). Earthquake ground motion characteristics and seismic energy dissipation. Earthquake Engineering and Structural Dynamics, 24, 1195-1213.

Uang, Ch. \& Bertero, V. (1990). Evaluation of seismic energy in structure. Earthquake Engineering and Structural Dynamics, 19, 77-90.

College of Engineering University of California al Berkeley (1988). Use of energy as a design criterion in earthquake-resistant design (Report UCB/EERC 88/18). Uang, Ch. \& Bertero, V.

\section{SOBRE LOS AUTORES}

M. Sc. Rubén Leandro Ulloa.

Magíster Scientaiae en Ingeniería Civil con énfasis en Estructuras, de la Universidad de Costa Rica, investigador independiente

Teléfonos: 392-7676 y 237-1570

Facsímil: 261-2382

Correo electrónico: rlulloa@cfia.or.cr;

ruben.leandro@amanco.com

Ph. D. Guillermo Santana Barboza.

Ingeniero civil, profesor catedrático e investigador de la Escuela de Ingeniería Civil, de la Universidad de Costa Rica.

Teléfono: 207-4012

Facsímil: 207-4440

Correo electrónico: santana@lanamme.ucr.ac.cr 
a)

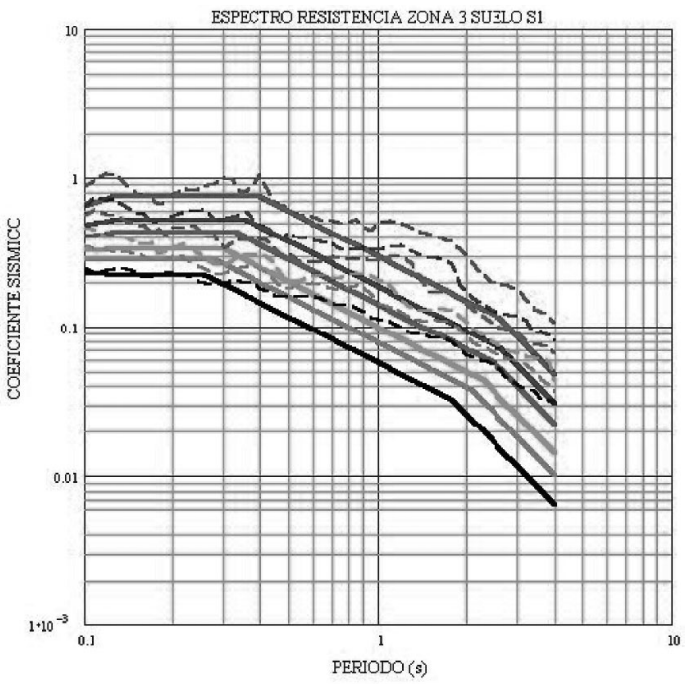

c)

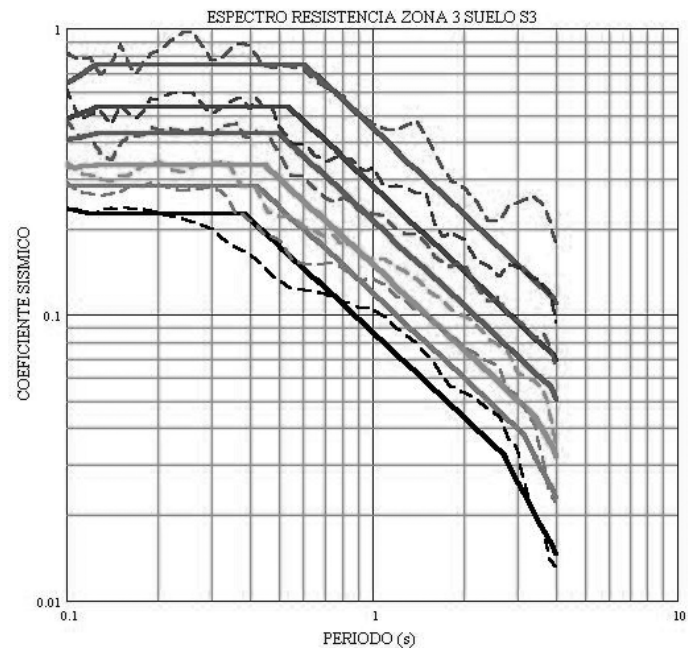

b)

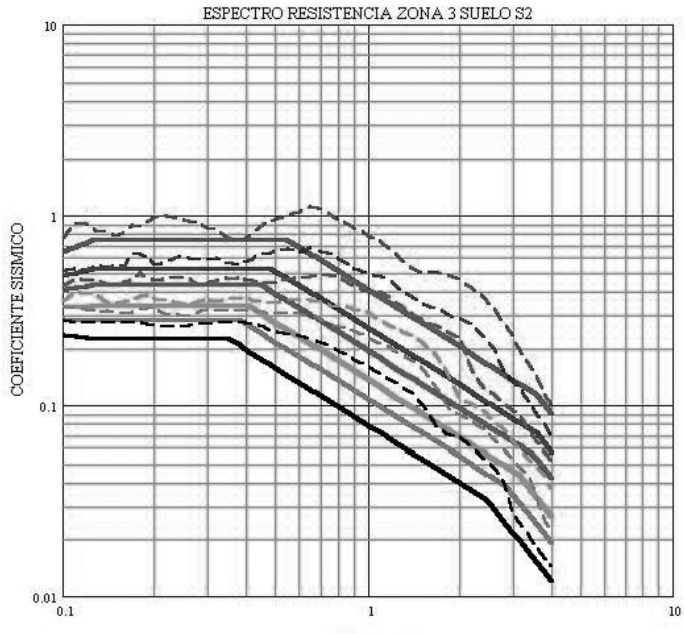

PERrODO (S)

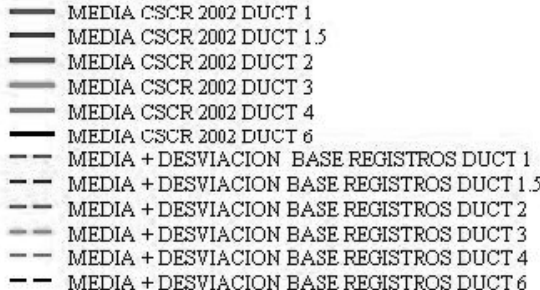

Figura 1. Comparación de espectro de diseño Area Metropolitana de San José Zona sísmica Z3 (Base de registros sísmicos versus CSCR-2002): a) Suelo S1, b) Suelo S2, Suelo S3.

Ver imágenes a color en la página 170. 
a)

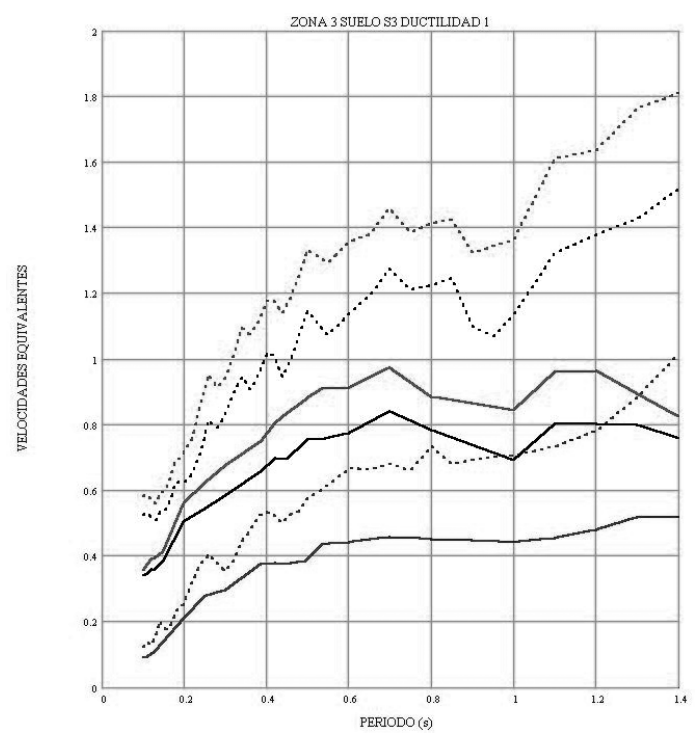

c)

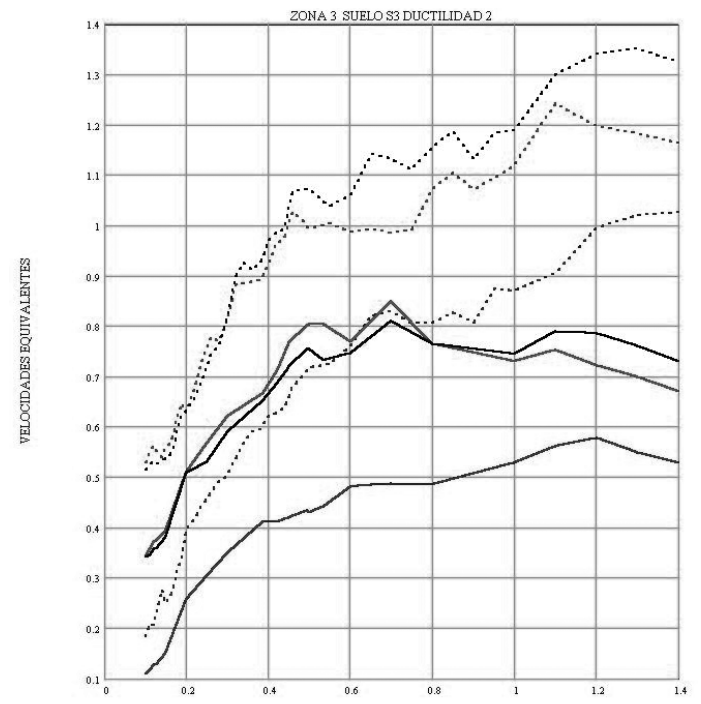

b)

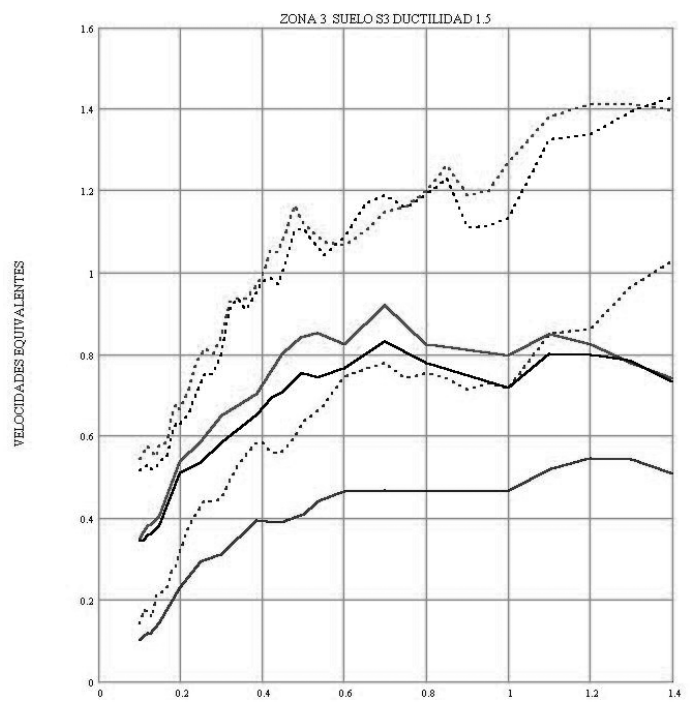

... PROMEDIO + DESVIACION BASE VELOC. EQUIVAL. ENERGIA DISIPADA .... PROMEDIO + DESVIACION BASE VELOC. EOUIVAL. ENERGIA ABSORBIDA $\cdots$ PROMEDIO + DESVIACION BASE VELOC. EQUIVAL. ENERGIA ENTRADA - PROMEDIO CSCR 2002 VELOC. EQUIVAL. ENERGIA DISIPADA - PROMEDIO CSCR 2002 VELOC. EOUIVAL. ENERGIA ABSORBIDA - PROMEDIO CSCR 2002 VELOC. EQUIVAL. ENERGIA ENTRADA

Figura 2a. Comparación de velocidades energéticas equivalentes Area Metropolitana Zon sísmica Z3 Suelo cimientación $\mathrm{S}_{3}$ (Base de registros sísmicos versus CSCR-2002): a) Ductilidad 1, b( Ductilidad 1.5, c) Ductilidad 2.

Ver imágenes a color en la página 171. 
a)

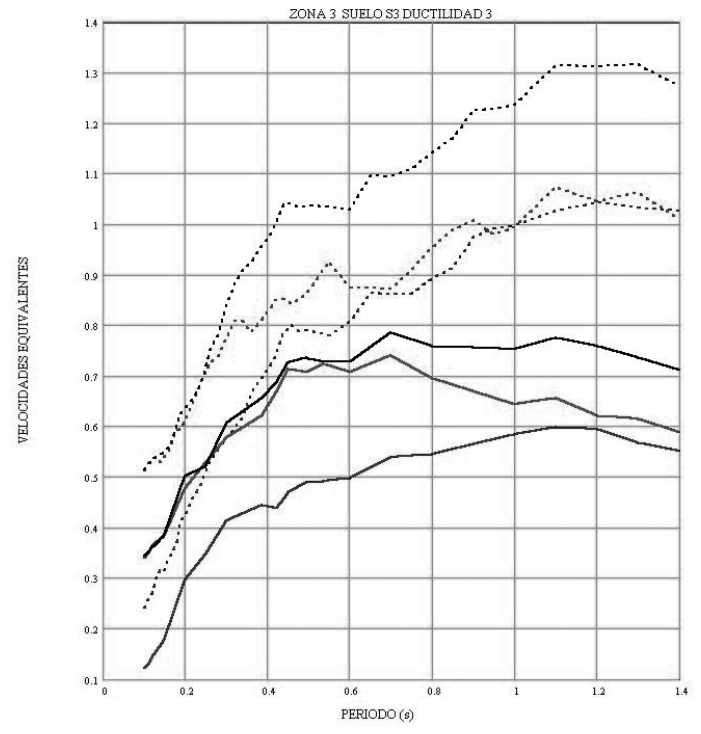

c)

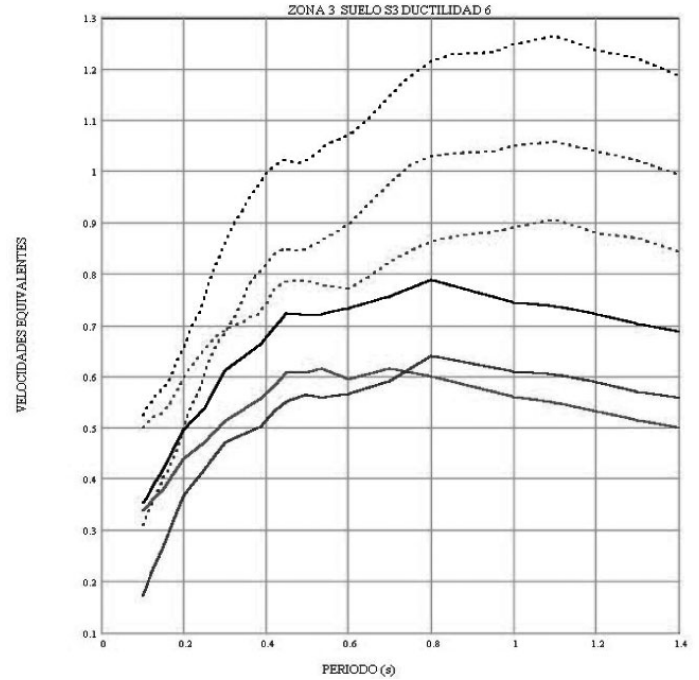

b)

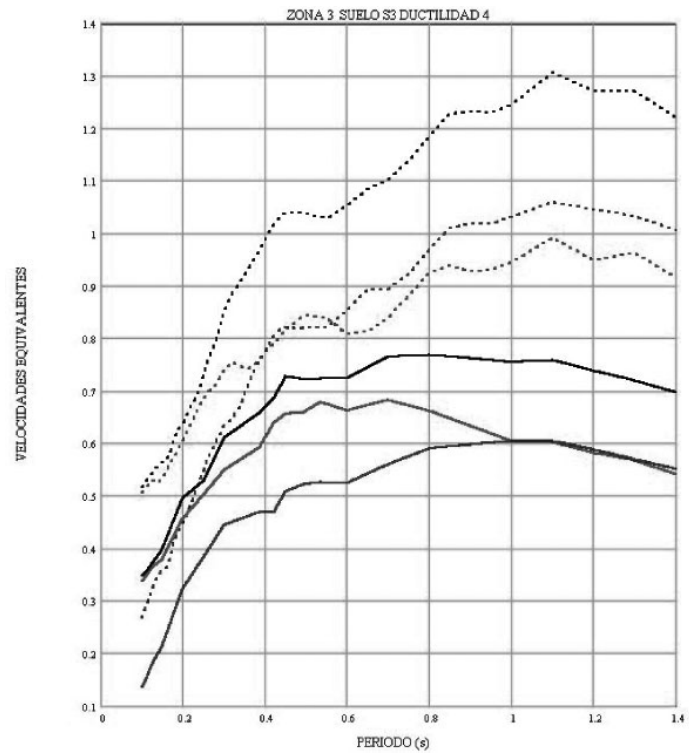

... PROMEDIO + DESVIACION BASE VELOC. EQUIVAL. ENERGIA DISIPADA .... PROMEDIO + DESVIACION BASE VELOC. EQUIVAL. ENERGIA ABSORBIDA $\cdots$ PROMEDIO + DESVIACION BASE VELOC. EQUIVAL. ENERGIA ENTRADA - PROMEDIO CSCR 2002 VELOC. EQUIVAL. ENERGIA DISIPADA

- PROMEDIO CSCR 2002 VELOC. EQUIVAL. ENERGIA ABSORBIDA

- PROMEDIO CSCR 2002 VELOC. EQUIVAL. ENERGIA ENTRADA

Figura 2b. Comparación de velocidades energéticas equivalentes Area Metropolitana Zon sísmica Z3 Suelo cimientación $\mathrm{S}_{3}$ (Base de registros sísmicos versus CSCR-2002): a) Ductilidad 3, b( Ductilidad 4, c) Ductilidad 6.

Ver imágenes a color en la página 172 
a)

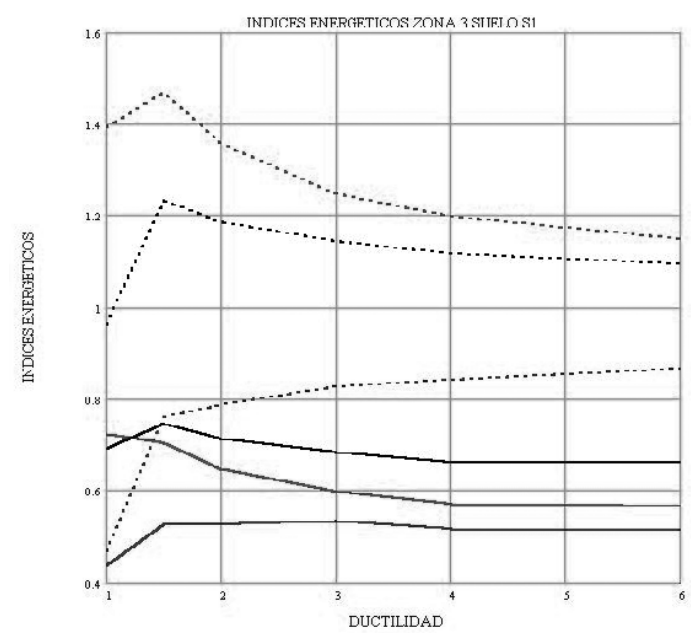

c)

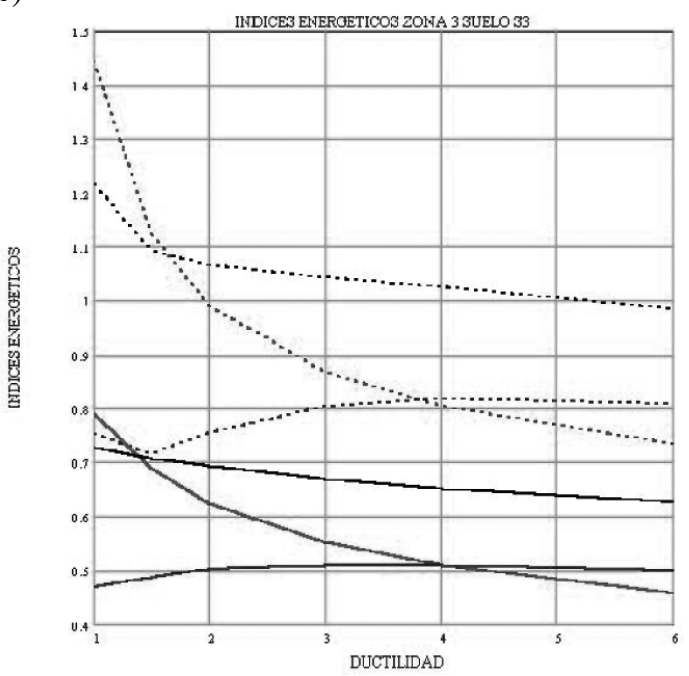

b)

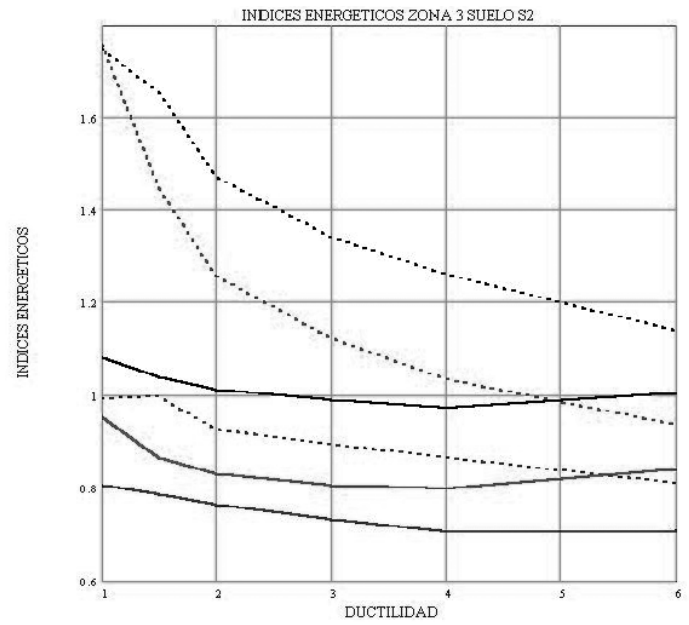

... PROMEDIO + DESVIACION BASE VELOC. EQUIVAL. ENERGIA DISIPADA

..- PROMEDIO + DESVIACION BASE VELOC. EQUIVAL. ENERGIA ABSORBIDA

... PROMEDIO + DESVIACION BASE VELOC. EQUIVAL. ENERGIA ENTRADA

- PROMEDIO CSCR 2002 VELOC. EQUIVAL. ENERGIA DISIPADA

— PROMEDIO CSCR 2002 VELOC. EQUIVAL. ENERGIA ABSORBIDA

Figura 3. Indices equivalentes energéticos Area Metropolitana Zona sísmica Z3 (BAse de registros sísmicos versus CSCR2002): a) Suelo S2, b) Suelo S2, c) Suelo S3.

Ver imágenes a color en la página 173. 